Pak. j. sci. ind. res. Ser. B: biol. sci. 201255 (1) 40-45

\title{
Anti Irritant Activity of Extract from the Aerial Parts of Echinops echinatus Compositae
}

\author{
Muhammad Zaheer $^{a *}$, Naqi Hussain ${ }^{\mathrm{a}}$, Salma Rahman ${ }^{\mathrm{a}}$ and Sajid-ur-Rehman ${ }^{\mathrm{b}}$ \\ ${ }^{a}$ Applied Chemistry Research Center, PCSIR Laboratories Complex, \\ Shahrah-e-Jalaluddin Roomi, Lahore-54600, Pakistan \\ ${ }^{b}$ Department of Pharmacy, The Islamia University of Bahawalpur, Bahawalpur, Pakistan
}

(received September 15, 2010; revised May 25, 2011; accepted August 2, 2011)

\begin{abstract}
Present study was focused on anti irritant potential of a xerophytic weed, Echinops echinatus. For this purpose, petroleum ether, chloroform and methanol extracts of various polarities from the aerial parts of E. echinatus were collected. Five fractions i.e., Ee-1 to Ee-5 were isolated from the chloroform extract of powdered plant by column and thin layer chromatography. The anti irritant potential of these fractions were evaluated on rabbit's skin. The anti irritant activity was evaluated from the healing mechanism on the abraded and irritated animal's skin. Two fractions (Ee-2 and Ee-4) appeared to be the potent anti irritant than others.
\end{abstract}

Keywords: Echinops echinatus, anti irritant activity, abraded, animal skin, chromatography

\section{Introduction}

Plants are considered to be biosynthetic laboratory not only for the synthesis of new chemical compounds but also for the synthesis complex of macro compounds (Adebanjo et al., 1983). The medicinal value of plants lies in chemical substances that produce a definite physiological action on human body. The most important bioactive compounds of plants are alkaloids, flavonoids, tannins and phenolic compounds (Edeoga et al., 2005). Earlier, it was thought that herbal drugs have no side effects but now it is observed that some of these drugs have undesirable effects (Abraham et al., 1986). Since disagreeable effects of various local plants and their products had not been explored, it was thus worth looking to evaluate such effects of locally occurring species like E. echinatus, its effect on the human and animal skin. The effect on skin was evaluated by contact dermatitis, an acute or chronic inflammation caused by external agents who have direct contact with the skin (Fregert, 1981; Marks and Samman, 1977). The adverse effects of plants and plant products on the skin can be categorized into different types but the most important is irritant contact dermatitis, a localized, superficial, exudative, non-immunological inflammation of the skin which is due to the direct influence of one or more external factors (Saeed, 1987; Stoner et al., 1983; Evans and Schmidt, 1980).

Many substances, including certain drugs and natural plant products on repeated contact with the skin may

*Author for correspondence; E-mail: mzaheerchem@yahoo.com cause dermatitis by the direct action (Saeed, 1987; Evans and Schmidt, 1980; Glickman, 1979). This occurs without previous sensitization and often immunological processes are not involved. During and after the first contact, no visual alterations may be observed, but after repeated contact, the skin gradually becomes erythmatous, drying and cracking occur and later on eczematous reaction with papules and vesicles may develop(Glickman, 1979; Marks and Samman, 1977). This type of contact dermatitis is known as irritant contact dermatitis (ICD) (Anderson et al., 1987; Saeed, 1987; Evans and Schmidt, 1980). The toxicity of the irritant compounds may vary with the season, stage of maturity and locality (Evans and Schmidt, 1979). Many plants causing irritant reaction are common house-hold plants or some time edible fruits may cause irritant reactions.

Echinops echinatus is a xerophytic weed commonly known as Untkatara growing in the desert places, in the plains and in the dry foot-hill areas of Indo-Pakistan, Iran and Hamalayas. The alkaloids, phytosterols, polyphenols saponins,and carotenoids in different parts of the plants were observed whilst anthraquinones were not detected (Hymete et al., 2005). Review of literature reveals estimation and isolation of various constituents such as flavonoids, acetylenic thiophenes, teraxasterol and teraxasterol acetates and a considerable number of pentacyclic triterpenoids and their esters (Singh and Pandey, 1990). Lipids and many alkaloids were also isolated and characterized (Hogale and Uthale, 1989; Chaudhuri, 1988; 1987). 
E. echinatus is found locally as weed in cultivated fields. Plant is known to have a variety of pharmacological activities. Folk medicines have described its use as an anti-inflammatory and wound healing plant but no scientific report of such use has been published so far. Since anti-inflammatory and wound healing property of E. echinatus has been described in literature (Singh et al., 1989), such property of this species diverted the dimensions of research work towards anti irritant activity. The main objective of this study was to evaluate anti irritant effects on animal skin by using different fractions of extracts that might be responsible for such activities.

\section{Materials and Methods}

E. echinatus plants were collected from different areas of Lahore (Pakistan) during the month of June, authenticated by the Department of Botany, Government College University, Lahore and deposited in the Lab. as GC Herb, Bot 604. The herbaceous plants were dried under shade at room temperature for 20 days.

Dried and pulverized aerial parts of E. echinatus (750 g), were extracted with methanol (2 liters) for $48 \mathrm{~h}$ and then solvent was evaporated using rotary evaporator (Heidolph Rotacool,Germany) at $45^{\circ} \mathrm{C}$. The obtained residue $5.2 \mathrm{~g}$ was extracted successively in petroleum ether $\left(40-60^{\circ} \mathrm{C}\right)$ and chloroform. Solvent of each extracted material was removed under reduced pressure using rotary evaporator; residues were weighed and exposed to UV rays for $24 \mathrm{~h}$ and checked for sterility on neutrient agar plates then refrigerated at $2-8{ }^{\circ} \mathrm{C}$ until further work was carried out (Nkere and Iroegbu, 2005).

Preliminary phytochemical analysis. Three extracts were evaluated for various classes of compounds using qualitative thin layer chromatography. The classes were identified by co-chromatography reference standards and spraying various colouring reagent onto TLC plates. Petroleum ether extract contained mainly lipids; chloroform extract indicated the presence of alkaloids and steroids by using Dragendorff's reagent and Libermann Burchard reagent, respectively.

Column chromatography. A glass column of $89 \times 1.5 \mathrm{~cm}$ was packed with $240 \mathrm{~g}$ of silica gel 60 (70-230 mesh), suspended in petroleum ether (Rahman et al., 2004). Chloroform extract (12 g) dissolved in $10 \mathrm{~mL}$ of chloroform was placed on the top of the column. The column was first eluted with $200 \mathrm{~mL}$ petroleum ether. Then the polarity of the system was changed by increasing the quantity of chloroform in petroleum ether. Each solvent ratios of frac-tions having similar spots were pooled together after monitoring with thin layer chromatography and bioassay was performed.

Biological assay for anti irritancy. A $20 \mathrm{mg}$ of the dried extract/column fraction was accurately weighed and made up to $20 \mathrm{mg} / 10 \mathrm{~mL}$ (w/v) solution with acetone. The dilution series were prepared according to the method of Evans and Schmidt (1979).

Experimental animals used in this study were male albino rabbits (Oryctolagus cuniculus), weighing 1-1.2 kg, kept under semiconstant temperature conditions and fed on animal fodder provided with tap water ad libitum. Sand paper with fine particles was used to irritate inner surface of rabbit's ear by rubbing it clock wise. This procedure was repeated 10 times until irritated area became red and erythma was produced which was measured in $2 \mathrm{~cm}$ diameter. For evaluation of anti irritant activity $100 \mu \mathrm{g}$ and $200 \mu \mathrm{g}$ solution from each solvent extracts/column fractions was applied to the irritated area. The ears were examined for intensity of erythma after every 30 min upto seven observations then five observations were recorded after every one hour. The animals were also examined after 24, 48 and $72 \mathrm{~h}$ to ascertain the chronic anti irritant dose.

A group of 4 rabbits was used for each dilution i.e., $100 \mu \mathrm{g} / 10 \mathrm{~mL}$ (group A) and $200 \mu \mathrm{g} / 10 \mathrm{~mL}$ (group B) of the isolated compounds. One ear was used as control while other was used as treated ear. Animals of the group B were also treated similarly by increasing concentration of each compound extract. Rabbits were examined after 30 min intervals for seven readings while after seven readings, they were observed after one hour interval in a similar way as in pilot assay. Chronic response was also recorded after 24, 48 and $72 \mathrm{~h}$ in a similar manner as in pilot assay. The number of ears showing marked healing areas was recorded. $\mathrm{AID}_{50}$ in $50 \%$ individuals was taken as a dose corresponding to $50 \%$ cumulative frequency. The anti irritant dose was expressed in $\mu \mathrm{g} / \mathrm{mL}$ per ear.

\section{Results and Discussion}

The percentage yields of the petroleum ether, chloroform and residual methanol extracts obtained from the whole herbs of E. echinatus was $0.61,1.60$ and $1.93 \%$, respectively.

All three extracts (as whole) were evaluated for their anti irritant activity on rabbits as experimental animals 
and results with $100 \mathrm{mg} / 10 \mathrm{~mL}$ and $200 \mathrm{mg} / 10 \mathrm{~mL}$ are shown in Tables 1 and 2, respectively.

The chloroform extract was further fractionated using varying ratios of petroleum ether chloroform and methanol (95:05:01 to 60:40:20) and chloroform methanol (90:07 to 50:50). About 30 fractions were obtained out of which 11 major fractions were evaluated for anti irritant activity as shown in Table 3 . The $R_{f}$ values of eleven column fractions of chloroform extract of E. echinatus obtained by thin layer chromatography has been shown in Table 4. Five major fractions of E. echinatus (Ee) namely Ee-1, Ee-2, Ee-3, Ee-4, and Ee-5 showed more activity than rest of the fractions and their anti irritant effect is shown in Table 5 and 6.

Extraction of phytochemical compounds from the aerial parts of plant was carried out using non-polar and polar solvents i.e. petroleum ether (40-60 c), chloroform and methanol. The residual methanol fraction was higher in yield than others. The components with intermediate polarity extracted with chloroform were next in yield. The non- polar components extracted in petroleum ether were in lowest yield. It could thus be concluded that E. echinatus contained a larger proportion of polar compounds and compounds of intermediate polarity than the non-polar components.

Irritancy assay was performed with all three types of solvent extracts of E. echinatus on rabbit's ears by the method of Hecker (Evans and Schmidt, 1979; Brain and Turner, 1975). The results indicate that the petroleum ether, chloroform and residual methanol extracts (as whole) did not exhibit irritant effects when the doses of $100 \mu \mathrm{g} / \mathrm{mL}$ and $200 \mu \mathrm{g} / \mathrm{mL}$ were applied on rabbit's ear. Anti irritant assay was also performed with all three types of solvent extracts/isolated compounds of E. echinatus on irritated rabbit's ear. The wound healing intensity produced by these extracts was evaluated by

Table 1. Anti irritant response of solvent extracts of E. echinatus on rabbit's ears

\begin{tabular}{|c|c|c|c|c|c|c|c|c|c|c|c|c|c|c|c|}
\hline \multirow{2}{*}{$\begin{array}{l}\text { Solvent fraction } \\
\text { Dose } \\
\mu \mathrm{g} / \mu \mathrm{L} / \mathrm{ear}\end{array}$} & \multicolumn{12}{|c|}{ Acute response(healed area in $\mathrm{cm}$ )* } & \multicolumn{3}{|c|}{ Chronic response $(\mathrm{cm})$} \\
\hline & $30 \mathrm{~min}$ & $1 \mathrm{~h}$ & $1.5 \mathrm{~h}$ & $2 \mathrm{~h}$ & $2.5 \mathrm{~h}$ & $3 \mathrm{~h}$ & $3.5 \mathrm{~h}$ & $4 \mathrm{~h}$ & $5 \mathrm{~h}$ & $6 \mathrm{~h}$ & $7 \mathrm{~h}$ & $8 \mathrm{~h}$ & $24 \mathrm{~h}$ & $48 \mathrm{~h}$ & $72 \mathrm{~h}$ \\
\hline $\begin{array}{l}\text { Chloroform } \\
100 \mu \mathrm{g} / 10 \mathrm{~mL}\end{array}$ & - & - & - & - & - & - & - & - & $\begin{array}{l}0.2 \\
\pm 0.04\end{array}$ & $\begin{array}{l}0.2 \\
\pm 0.04\end{array}$ & $\begin{array}{l}0.2 \\
\pm 0.04\end{array}$ & $\begin{array}{l}0.3 \\
\pm 0.04\end{array}$ & $\begin{array}{l}0.4 \\
\pm 0.04\end{array}$ & $\begin{array}{l}0.6 \\
\pm 0.04\end{array}$ & $\begin{array}{l}0.8 \\
\pm 0.04\end{array}$ \\
\hline $\begin{array}{l}\text { Methanol } \\
100 \mu \mathrm{g} / 10 \mathrm{~mL}\end{array}$ & - & - & - & - & - & - & - & - & - & $\begin{array}{l}0.2 \\
\pm 0.04\end{array}$ & $\begin{array}{l}0.2 \\
\pm 0.04\end{array}$ & $\begin{array}{l}0.3 \\
\pm 0.04\end{array}$ & $\begin{array}{l}0.4 \\
\pm 0.04\end{array}$ & $\begin{array}{l}0.6 \\
\pm 0.04\end{array}$ & $\begin{array}{l}0.8 \\
\pm 0.04\end{array}$ \\
\hline $\begin{array}{l}\text { Petroleum ether } \\
100 \mu \mathrm{g} / 10 \mathrm{~mL}\end{array}$ & - & - & - & - & - & - & - & - & - & - & - & $\begin{array}{l}0.2 \\
\pm 0.04\end{array}$ & $\begin{array}{l}0.3 \\
\pm 0.04\end{array}$ & $\begin{array}{l}0.3 \\
\pm 0.04\end{array}$ & $\begin{array}{l}0.3 \\
\pm 0.04\end{array}$ \\
\hline $\begin{array}{l}\text { Control } \\
\text { (untreated) }\end{array}$ & - & - & - & - & - & - & - & - & - & - & - & - & - & - & - \\
\hline
\end{tabular}

* = mean reading of healing area replicate; $\mathrm{h}=$ hours of healing.

Table 2. Anti irritant response of solvent extracts of E. echinatus on rabbit's ears

\begin{tabular}{|c|c|c|c|c|c|c|c|c|c|c|c|c|c|c|c|}
\hline \multirow{2}{*}{$\begin{array}{l}\text { Solvent fraction } \\
\text { Dose } \\
\mu \mathrm{g} / \mu \mathrm{L} / \mathrm{ear}\end{array}$} & \multicolumn{12}{|c|}{ Acute response(healed area in $\mathrm{cm}$ )* } & \multicolumn{3}{|c|}{ Chronic response $(\mathrm{cm})$} \\
\hline & $30 \mathrm{~min}$ & $1 \mathrm{~h}$ & $1.5 \mathrm{~h}$ & $2 \mathrm{~h}$ & $2.5 \mathrm{~h}$ & $3 \mathrm{~h}$ & $3.5 \mathrm{~h}$ & $4 \mathrm{~h}$ & $5 \mathrm{~h}$ & $6 \mathrm{~h}$ & $7 \mathrm{~h}$ & $8 \mathrm{~h}$ & $24 \mathrm{~h}$ & $48 \mathrm{~h}$ & $72 \mathrm{~h}$ \\
\hline $\begin{array}{l}\text { Chloroform } \\
200 \mu \mathrm{g} / 10 \mathrm{~mL}\end{array}$ & - & - & - & - & $\begin{array}{l}0.2 \\
\pm 0.04\end{array}$ & $\begin{array}{l}0.3 \\
\pm 0.04\end{array}$ & $\begin{array}{l}0.4 \\
\pm 0.02\end{array}$ & $\begin{array}{l}0.5 \\
\pm 0.02\end{array}$ & $\begin{array}{l}0.6 \\
\pm 0.04\end{array}$ & $\begin{array}{l}0.7 \\
\pm 0.04\end{array}$ & $\begin{array}{l}0.8 \\
\pm 0.04\end{array}$ & $\begin{array}{l}0.9 \\
\pm 0.02\end{array}$ & $\begin{array}{l}1.2 \\
\pm 0.04\end{array}$ & $\begin{array}{l}1.6 \\
\pm 0.02\end{array}$ & $\begin{array}{l}1.9 \\
\pm 0.02\end{array}$ \\
\hline $\begin{array}{l}\text { Methanol } \\
200 \mu \mathrm{g} / 10 \mathrm{~mL}\end{array}$ & - & - & - & - & - & $\begin{array}{l}0.2 \\
\pm 0.04\end{array}$ & $\begin{array}{l}0.3 \\
\pm 0.04\end{array}$ & $\begin{array}{l}0.4 \\
\pm 0.04\end{array}$ & $\begin{array}{l}0.5 \\
\pm 0.04\end{array}$ & $\begin{array}{l}0.6 \\
\pm 0.04\end{array}$ & $\begin{array}{l}0.7 \\
\pm 0.04\end{array}$ & $\begin{array}{l}0.8 \\
\pm 0.04\end{array}$ & $\begin{array}{l}1.1 \\
\pm 0.04\end{array}$ & $\begin{array}{l}1.5 \\
\pm 0.02\end{array}$ & $\begin{array}{l}1.8 \\
\pm 0.02\end{array}$ \\
\hline $\begin{array}{l}\text { Petroleum ether } \\
200 \mu \mathrm{g} / 10 \mathrm{~mL}\end{array}$ & - & - & - & - & - & - & - & - & - & $\begin{array}{l}0.2 \\
\pm 0.04\end{array}$ & $\begin{array}{l}0.3 \\
\pm 0.04\end{array}$ & $\begin{array}{l}0.4 \\
\pm 0.04\end{array}$ & $\begin{array}{l}0.7 \\
\pm 0.04\end{array}$ & $\begin{array}{l}1.0 \\
\pm 0.04\end{array}$ & $\begin{array}{l}1.4 \\
\pm 0.04\end{array}$ \\
\hline $\begin{array}{l}\text { Control } \\
\text { (untreated) }\end{array}$ & - & - & - & - & - & - & - & - & - & - & - & - & - & - & - \\
\hline
\end{tabular}

* $=$ mean reading of healing area replicate; $\mathrm{h}=$ hours of healing. 
Table 3. Anti irritant response of the pooled fractions of aerial parts of E. echinatus on rabbit's ear

\begin{tabular}{|c|c|c|c|c|c|c|c|c|c|c|c|c|c|}
\hline \multirow{2}{*}{$\begin{array}{l}\text { Frac- } \\
\text { tions }\end{array}$} & \multicolumn{13}{|c|}{ Anti irritant response (healed areas in $\mathrm{cm}$ )* } \\
\hline & $1 \mathrm{~h}$ & $2 \mathrm{~h}$ & $2.5 \mathrm{~h}$ & $3 \mathrm{~h}$ & $3.5 \mathrm{~h}$ & $4 \mathrm{~h}$ & $5 \mathrm{~h}$ & $6 \mathrm{~h}$ & $7 \mathrm{~h}$ & $8 \mathrm{~h}$ & $24 \mathrm{~h}$ & $48 \mathrm{~h}$ & $72 \mathrm{~h}$ \\
\hline E1 & - & - & - & - & - & - & - & - & $\begin{array}{l}0.2 \\
\pm 0.04\end{array}$ & $\begin{array}{l}0.3 \\
\pm 0.04\end{array}$ & $\begin{array}{l}0.5 \\
\pm 0.04\end{array}$ & $\begin{array}{l}0.7 \\
\pm 0.02\end{array}$ & $\begin{array}{l}0.9 \\
\pm 0.02\end{array}$ \\
\hline E2 & - & - & - & - & - & $\begin{array}{l}0.2 \\
\pm 0.02\end{array}$ & $\begin{array}{l}0.2 \\
\pm 0.04\end{array}$ & $\begin{array}{l}0.3 \\
\pm 0.04\end{array}$ & $\begin{array}{l}0.4 \\
\pm 0.02\end{array}$ & $\begin{array}{l}0.5 \\
\pm 0.04\end{array}$ & $\begin{array}{l}0.8 \\
\pm 0.02\end{array}$ & $\begin{array}{l}1.2 \\
\pm 0.02\end{array}$ & $\begin{array}{l}1.7 \\
\pm 0.02\end{array}$ \\
\hline E3 & - & - & - & - & - & - & $\begin{array}{l}0.2 \\
\pm 0.04\end{array}$ & $\begin{array}{l}0.3 \\
\pm 0.02\end{array}$ & $\begin{array}{l}0.4 \\
\pm 0.02\end{array}$ & $\begin{array}{l}0.5 \\
\pm 0.04\end{array}$ & $\begin{array}{l}0.7 \\
\pm 0.04\end{array}$ & $\begin{array}{l}1.1 \\
\pm 0.02\end{array}$ & $\begin{array}{l}1.6 \\
\pm 0.02\end{array}$ \\
\hline E4 & - & - & - & - & - & - & - & - & - & $\begin{array}{l}0.2 \\
\pm 0.04\end{array}$ & $\begin{array}{l}0.4 \\
\pm 0.04\end{array}$ & $\begin{array}{l}0.6 \\
\pm 0.02\end{array}$ & $\begin{array}{l}0.8 \\
\pm 0.02\end{array}$ \\
\hline E5 & - & - & - & - & $\begin{array}{l}0.2 \\
\pm 0.04\end{array}$ & $\begin{array}{l}0.3 \\
\pm 0.04\end{array}$ & $\begin{array}{l}0.4 \\
\pm 0.02\end{array}$ & $\begin{array}{l}0.5 \\
\pm 0.04\end{array}$ & $\begin{array}{l}0.6 \\
\pm 0.02\end{array}$ & $\begin{array}{l}0.7 \\
\pm 0.04\end{array}$ & $\begin{array}{l}0.9 \\
\pm 0.02\end{array}$ & $\begin{array}{l}1.2 \\
\pm 0.04\end{array}$ & $\begin{array}{l}1.5 \\
\pm 0.02\end{array}$ \\
\hline E6 & - & - & - & - & - & - & - & - & - & - & $\begin{array}{l}0.2 \\
\pm 0.04\end{array}$ & $\begin{array}{l}0.2 \\
\pm 0.02\end{array}$ & $\begin{array}{l}0.3 \\
\pm 0.02\end{array}$ \\
\hline E7 & - & - & - & - & - & - & - & - & - & $\begin{array}{l}0.2 \\
\pm 0.04\end{array}$ & $\begin{array}{l}0.2 \\
\pm 0.02\end{array}$ & $\begin{array}{l}0.3 \\
\pm 0.04\end{array}$ & $\begin{array}{l}0.4 \\
\pm 0.04\end{array}$ \\
\hline E8 & - & - & - & - & - & $\begin{array}{l}0.2 \\
\pm 0.04\end{array}$ & $\begin{array}{l}0.3 \\
\pm 0.04\end{array}$ & $\begin{array}{l}0.4 \\
\pm 0.04\end{array}$ & $\begin{array}{l}0.5 \\
\pm 0.02\end{array}$ & $\begin{array}{l}0.6 \\
\pm 0.02\end{array}$ & $\begin{array}{l}0.8 \\
\pm 0.04\end{array}$ & $\begin{array}{l}1.0 \\
\pm 0.02\end{array}$ & $\begin{array}{l}1.3 \\
\pm 0.04\end{array}$ \\
\hline E9 & - & - & - & - & - & $\begin{array}{l}0.2 \\
\pm 0.04\end{array}$ & $\begin{array}{l}0.3 \\
\pm 0.04\end{array}$ & $\begin{array}{l}0.4 \\
\pm 0.04\end{array}$ & $\begin{array}{l}0.5 \\
\pm 0.02\end{array}$ & $\begin{array}{l}0.6 \\
\pm 0.04\end{array}$ & $\begin{array}{l}0.8 \\
\pm 0.02\end{array}$ & $\begin{array}{l}1.1 \\
\pm 0.04\end{array}$ & $\begin{array}{l}1.4 \\
\pm 0.02\end{array}$ \\
\hline E10 & - & - & - & - & - & - & - & $\begin{array}{l}0.2 \\
\pm 0.04\end{array}$ & $\begin{array}{l}0.3 \\
\pm 0.02\end{array}$ & $\begin{array}{l}0.4 \\
\pm 0.04\end{array}$ & $\begin{array}{l}0.6 \\
\pm 0.04\end{array}$ & $\begin{array}{l}0.7 \\
\pm 0.04\end{array}$ & $\begin{array}{l}0.9 \\
\pm 0.02\end{array}$ \\
\hline E11 & - & - & - & - & - & - & - & - & - & - & - & $\begin{array}{l}0.2 \\
\pm 0.02\end{array}$ & $\begin{array}{l}0.2 \\
\pm 0.02\end{array}$ \\
\hline
\end{tabular}

* = mean reading of healing area replicate; $\mathrm{h}=$ hours of healing.

Table 4. Comparative thin layer chromatographic analysis of pooled column fractions of chloroform extracts of E. echinatus

\begin{tabular}{llll}
\hline $\begin{array}{l}\text { Pooled } \\
\text { fraction }\end{array}$ & Eluting solvent & $\begin{array}{l}\text { No. of } \\
\text { compound }\end{array}$ & $\mathrm{R}_{\mathrm{f} \text { values }}$ \\
\hline 1. & $\mathrm{PE}(100 \%)$ & 1 & 78 \\
2. & $\mathrm{PE}(100 \%)$ & 1 & 65 \\
3. & $\mathrm{PE} / \mathrm{CHCl}_{3}(95: 05)$ & 1 & 84 \\
4. & $\mathrm{PE} / \mathrm{CHCl}_{3}(90: 10)$ & 1 & 75 \\
5. & $\mathrm{PE} / \mathrm{CHCl}_{3}(90: 10)$ & 1 & 68 \\
6. & $\mathrm{PE} / \mathrm{CHCl}_{3}(80: 20)$ & 1 & 80 \\
7. & $\mathrm{PE} / \mathrm{CHCl}_{3}(80: 20)$ & 2 & 24,82 \\
8. & $\mathrm{PE} / \mathrm{CHCl}_{3}(80: 20)$ & 1 & 68 \\
9. & $\mathrm{PE} / \mathrm{CHCl}_{3}(80: 30)$ & 1 & 79 \\
10. & $\mathrm{PE} / \mathrm{CHCl}_{3}(80: 30)$ & 2 & 50,81 \\
11. & $\mathrm{PE} / \mathrm{CHCl}_{3}(80: 30)$ & 2 & 68,59 \\
\hline \hline $\mathrm{PE}$
\end{tabular}

$\mathrm{PE}=$ petroleum ether. healed area. Petroleum ether extract did not show any significant anti irritant response. Chloroform extract showed well marked wound healing property under the influence of $200 \mu \mathrm{g} / 10 \mathrm{~mL}$ on irritated rabbit's ear. Residual methanol extract showed weak anti irritant response. It could thus be concluded that the polar and intermediate polar constituents of E. echinatus were responsible for its anti irritant and anti-inflam-matory property on irritated animal skin.

Since the chloroform extract of E. echinatus was active and obtained in sufficient amount, it was further subjected to column chromatography to isolate the active compounds using an increasing quantity of chloroform in petroleum ether. Eleven fractions were obtained as shown in Table 3. Anti irritant response of these eleven fractions were evaluated, as shown in Table 5. Five pooled column fractions out of eleven 
Table 5. Anti irritant response of the isolated compounds Ee-1 to Ee-5 from chloroform extracts of aerial parts of E. echinatus on rabbit's ears (Dose $=100 \mu \mathrm{g} / 10 \mathrm{~mL}$ )

\begin{tabular}{|c|c|c|c|c|c|c|c|c|c|c|c|c|c|c|c|}
\hline \multirow{2}{*}{$\begin{array}{l}\text { Com- } \\
\text { pound }\end{array}$} & \multicolumn{15}{|c|}{ Anti irritant response(healed areas in $\mathrm{cm}$ )* } \\
\hline & $30 \mathrm{~min}$ & $1 \mathrm{~h}$ & $1.5 \mathrm{~h}$ & $2 \mathrm{~h}$ & $2.5 \mathrm{~h}$ & $3 \mathrm{~h}$ & $3.5 \mathrm{~h}$ & $4 \mathrm{~h}$ & $5 \mathrm{~h}$ & $6 \mathrm{~h}$ & $7 \mathrm{~h}$ & $8 \mathrm{~h}$ & $24 \mathrm{~h}$ & $48 \mathrm{~h}$ & $72 \mathrm{~h}$ \\
\hline Ee-1 & - & - & - & - & - & - & - & $\begin{array}{l}0.2 \\
\pm 0.04\end{array}$ & $\begin{array}{l}0.2 \\
\pm 0.02\end{array}$ & $\begin{array}{l}0.3 \\
\pm 0.04\end{array}$ & $\begin{array}{l}0.4 \\
\pm 0.04\end{array}$ & $\begin{array}{l}0.5 \\
\pm 0.04\end{array}$ & $\begin{array}{l}0.8 \\
\pm 0.02\end{array}$ & $\begin{array}{l}1.0 \\
\pm 0.02\end{array}$ & $\begin{array}{l}1.2 \\
\pm 0.02\end{array}$ \\
\hline Ee-2 & - & - & - & - & - & - & $\begin{array}{l}0.2 \\
\pm 0.02\end{array}$ & $\begin{array}{l}0.3 \\
\pm 0.04\end{array}$ & $\begin{array}{l}0.4 \\
\pm 0.02\end{array}$ & $\begin{array}{l}0.4 \\
\pm 0.04\end{array}$ & $\begin{array}{l}0.5 \\
\pm 0.04\end{array}$ & $\begin{array}{l}0.6 \\
\pm 0.02\end{array}$ & $\begin{array}{l}0.9 \\
\pm 0.02\end{array}$ & $\begin{array}{l}1.1 \\
\pm 0.02\end{array}$ & $\begin{array}{l}1.5 \\
\pm 0.02\end{array}$ \\
\hline Ee-3 & - & - & - & - & - & - & - & - & $\begin{array}{l}0.2 \\
\pm 0.02\end{array}$ & $\begin{array}{l}0.3 \\
\pm 0.02\end{array}$ & $\begin{array}{l}0.3 \\
\pm 0.02\end{array}$ & $\begin{array}{l}0.4 \\
\pm 0.02\end{array}$ & $\begin{array}{l}0.6 \\
\pm 0.02\end{array}$ & $\begin{array}{l}0.8 \\
\pm 0.02\end{array}$ & $\begin{array}{l}1.1 \\
\pm 0.02\end{array}$ \\
\hline Ee-4 & - & - & - & - & - & - & - & $\begin{array}{l}0.2 \\
\pm 0.04\end{array}$ & $\begin{array}{l}0.3 \\
\pm 0.04\end{array}$ & $\begin{array}{l}0.4 \\
\pm 0.02\end{array}$ & $\begin{array}{l}0.5 \\
\pm 0.02\end{array}$ & $\begin{array}{l}0.6 \\
\pm 0.04\end{array}$ & $\begin{array}{l}0.9 \\
\pm 0.02\end{array}$ & $\begin{array}{l}1.2 \\
\pm 0.02\end{array}$ & $\begin{array}{l}1.6 \\
\pm 0.02\end{array}$ \\
\hline Ee-5 & - & - & - & - & - & - & - & - & - & $\begin{array}{l}0.2 \\
\pm 0.04\end{array}$ & $\begin{array}{l}0.3 \\
\pm 0.02\end{array}$ & $\begin{array}{l}0.4 \\
\pm 0.04\end{array}$ & $\begin{array}{l}0.6 \\
\pm 0.04\end{array}$ & $\begin{array}{l}0.8 \\
\pm 0.02\end{array}$ & $\begin{array}{l}1.1 \\
\pm 0.02\end{array}$ \\
\hline
\end{tabular}

* = mean reading of healing area replicates; $\mathrm{h}$ = hours of healing; Ee $=$ E. echinatus

Table 6. Anti irritant response of the isolated compounds Ee-1 to Ee-5 from chloroform extracts of aerial parts of E. echinatus on rabbit's ears (Dose $=200 \mu \mathrm{g} / 10 \mathrm{~mL}$ )

\begin{tabular}{|c|c|c|c|c|c|c|c|c|c|c|c|c|c|c|c|}
\hline \multirow{2}{*}{$\begin{array}{l}\text { Com- } \\
\text { pound }\end{array}$} & \multicolumn{15}{|c|}{ Anti irritant response (healed areas in $\mathrm{cm}$ )*jk } \\
\hline & $30 \mathrm{~min}$ & $1 \mathrm{~h}$ & $1.5 \mathrm{~h}$ & $2 \mathrm{~h}$ & $2.5 \mathrm{~h}$ & $3 \mathrm{~h}$ & $3.5 \mathrm{~h}$ & $4 \mathrm{~h}$ & $5 \mathrm{~h}$ & $6 \mathrm{~h}$ & $7 \mathrm{~h}$ & $8 \mathrm{~h}$ & $24 \mathrm{~h}$ & $48 \mathrm{~h}$ & $72 \mathrm{~h}$ \\
\hline Ee-1 & - & - & - & - & - & - & $\begin{array}{l}0.2 \\
\pm 0.04\end{array}$ & $\begin{array}{l}0.3 \\
\pm 0.02\end{array}$ & $\begin{array}{l}0.4 \\
\pm 0.04\end{array}$ & $\begin{array}{l}0.5 \\
\pm 0.02\end{array}$ & $\begin{array}{l}0.6 \\
\pm 0.04\end{array}$ & $\begin{array}{l}0.7 \\
\pm 0.02\end{array}$ & $\begin{array}{l}1.0 \\
\pm 0.02\end{array}$ & $\begin{array}{l}1.4 \\
\pm 0.02\end{array}$ & $\begin{array}{l}1.6 \\
\pm 0.02\end{array}$ \\
\hline Ee-2 & - & - & - & - & $\begin{array}{l}0.2 \\
\pm 0.04\end{array}$ & $\begin{array}{l}0.3 \\
\pm 0.02\end{array}$ & $\begin{array}{l}0.4 \\
\pm 0.04\end{array}$ & $\begin{array}{l}0.5 \\
\pm 0.04\end{array}$ & $\begin{array}{l}0.7 \\
\pm 0.04\end{array}$ & $\begin{array}{l}0.8 \\
\pm 0.02\end{array}$ & $\begin{array}{l}1.0 \\
\pm 0.04\end{array}$ & $\begin{array}{l}1.2 \\
\pm 0.02\end{array}$ & $\begin{array}{l}1.6 \\
\pm 0.02\end{array}$ & $\begin{array}{l}1.9 \\
\pm 0.02\end{array}$ & healed \\
\hline Ee-3 & - & - & - & - & - & - & - & - & $\begin{array}{l}0.2 \\
\pm 0.04\end{array}$ & $\begin{array}{l}0.3 \\
\pm 0.04\end{array}$ & $\begin{array}{l}0.4 \\
\pm 0.02\end{array}$ & $\begin{array}{l}0.6 \\
\pm 0.02\end{array}$ & $\begin{array}{l}0.9 \\
\pm 0.02\end{array}$ & $\begin{array}{l}1.3 \\
\pm 0.02\end{array}$ & $\begin{array}{l}1.5 \\
\pm 0.02\end{array}$ \\
\hline Ee-4 & - & - & - & - & $\begin{array}{l}0.2 \\
\pm 0.04\end{array}$ & $\begin{array}{l}0.3 \\
\pm 0.04\end{array}$ & $\begin{array}{l}0.4 \\
\pm 0.04\end{array}$ & $\begin{array}{l}0.6 \\
\pm 0.02\end{array}$ & $\begin{array}{l}0.7 \\
\pm 0.02\end{array}$ & $\begin{array}{l}0.8 \\
\pm 0.04\end{array}$ & $\begin{array}{l}0.9 \\
\pm 0.04\end{array}$ & $\begin{array}{l}1.0 \\
\pm 0.02\end{array}$ & $\begin{array}{l}1.4 \\
\pm 0.02\end{array}$ & $\begin{array}{l}1.8 \\
\pm 0.02\end{array}$ & healed \\
\hline Ee-5 & - & - & - & - & - & - & - & $\begin{array}{l}0.2 \\
\pm 0.02\end{array}$ & $\begin{array}{l}0.3 \\
\pm 0.04\end{array}$ & $\begin{array}{l}0.4 \\
\pm 0.04\end{array}$ & $\begin{array}{l}0.5 \\
\pm 0.04\end{array}$ & $\begin{array}{l}0.7 \\
\pm 0.04\end{array}$ & $\begin{array}{l}1.0 \\
\pm 0.02\end{array}$ & $\begin{array}{l}1.3 \\
\pm 0.02\end{array}$ & $\begin{array}{l}1.7 \\
\pm 0.02\end{array}$ \\
\hline
\end{tabular}

* = mean reading of healing area replicates; $\mathrm{h}=$ hours of healing; $\mathrm{Ee}=$ E. echinatus

were dermatologically more active. They produced well marked anti irritant responses on irritated rabbit's ear after 30 min of application and in most instances this reaction lasted for about $72 \mathrm{~h}$. Their anti irritant responses started after nearly $3 \mathrm{~h}$ and lasted for even more than $72 \mathrm{~h}$. Well marked anti irritant response healing intensity of irritated skin was observed.

The present study encourage the herbal practitioner for the rational use of medicinal plants to treat different skin diseases including skin irritancy as the plant used in this study has shown marked anti irritant activity. Anti inflammatory, antifungal activity and analgesic activity of this plant has been described (Singh et al., 2008; Fokialakis et al., 2006) and present study provides the first preliminary report on anti irritant effects of Echinops echinatus.

\section{References}

Abraham, Z., Bhakuni, D.S., Garg, H.S., Goel, A.K., Mehrotra, B.N., Patnaik, G.K. 1986. Screening of Indian plants for biological activity. Part 12. Indian Journal of Experimental Biology, 24: 48-68.

Adebanjo, A.O., Adiwumi, C.O., Essein, E.E. 1983. International Symposiun on Medicinal Plants. 152158, University of Ifoc, Nigeria.

Anderson, K.E., Benezra, C., Burrows, D., Camarasa, J., Gossen, A.D., Ducombs, G., Forsch, P., Lachepelle, J.M., Lathi, A., Menne, T., Raycroft, 
R., Scheper, R., White, I., Wlikimson. 1987. Contact dermatitis- A review. Contact Dermatitis, 16: 55-78.

Brain, K.R., Turner, T.D. 1975. The Practical Evaluation of Phytopharmaceuticals, 144 pp., Wriht-Scientechnica, Bristol, UK.

Chaudhuri, P.K. 1988. Constituents of flowers of Echinops echinatus. Fitoterapia, 59: 150-151.

Choudhuri, P.K. 1987. Echinozolinone, an alkaloid from Echinatus. Phytochemistry, 26: 587-589.

Edeoga, H.O., Okwu, D.E., Mbaeble, B.O. 2005. Phytochemical constituents of some Nigerian medicinal plants. African Journal of Biotechnology, 4: 685-688.

Evans, F.J., Schmidt, R.J. 1980. Plants and plant products that induce contact dermatitis- A review. Planta Medica, 38: 289-316.

Evans, F.J., Schmidt, R.J. 1979. An assay procedure for the comparative irritancy testing of esters in the tigliane and daphnane series. Inflammation, 3: 215-223.

Fokialakis, N., Cantrell, C.L., Duke, S.O., Skaltsounis, A.L., Wedge, D.E. 2006. Antifungal activity of thiophenes from Echinops ritro. Journal of Agriculture and Food Chemistry, 54: 1651-1655.

Fregert, S. 1981. Manual of Contact Dermatitis, pp. 910, $2^{\text {nd }}$ edition, Year-book Medical Publisher, Chicago, USA.

Glickman, F.S. 1979. General Dermatology, pp. 151152, P.S.G. Publishing Littleton, Massachustts, USA.

Hogale, M.B., Uthale, A.C. 1989. Chemical constituents of Echinops echinatus. Oriented Journal of Chemistry,
4: 334-335.

Hymete, A., Iversen, T.H., Rohloff, J., Erko, B. 2005. Screening of Echinops ellenbeckii and Echinops longisetus for biological activities and chemical constituents, Phytomedicine, 12: 675-679.

Marks, R., Samman, P.D. 1977. Dermatology, vol. 6, pp. 130-131, William Heinman Medical Books Ltd., London, UK.

Nkere, C.K., Iroegbu, C.U. 2005. Antibacterial screening of the root, seed and stem bark extracts of Picralima nitida. African Journal of Biotechnology, 4: 522-526.

Rahman, S., Chaudri, T.A., Iqbal, M.Z. 2004. Lipid classes of Hordeum vulgare. Pakistan Journal of Scientific and Industrial Research, 47: 303-308.

Saeed, M.A. 1987. Allergic Cross-sensitization to some Oleo-gum-resins in Colophony Sensitized Animals. Ph.D. Thesis, University of Wales, Cardiff, UK.

Singh, A., Malhotra, S., Subban, R. 2008. Anti inflammatory and analgesic agents from Indian medicinal plants. International Journal of Integrative Biology, 3: 1 - 57.

Singh, K.N., Pandey, V.B. 1990. Constituents of Echinops. Oriental Journal of Chemistry, 5: 342344.

Singh, B., Gambhir, S.S., Pandey, V.K., Josin, V.K., 1989. Anti inflammatory activity of Echinops echinatus. Journal of Ethnopharmacology, 25: 189199.

Stoner, J.G., Rasmussen, J.E. 1983. Plant dermatitis. Journal of American Academy of Dermatitis, 9: 1-15. 